\title{
Obstructive sleep apnea and excessive day time sleepiness as measured by epworth sleepiness scale
}

\author{
Tandon $S^{1}$, Joshi $P^{2}$, Nagdeote $S . T^{3}$ \\ ${ }^{1}$ Dr Sanjay Tandon, Professor and Head, ${ }^{2}$ Dr Priyanka Joshi, Post Graduate Student, ${ }^{3}$ Dr S. T. Nagdeote, Professor, All \\ are affiliated with Department of Pulmonary Medicine, Peoples College of Medical Science And Research Centre \\ Bhopal.
}

Address for Correspondence: Email- pulmedph@gmail.com, drvijaypriya14@gmail.com

\begin{abstract}
Introduction: Many patients search for medical care for symptom of a sleep disorder, obstructive sleep apnea hypopnea syndrome (OSAHS). Currently overnight polysomnography is the gold standard for diagnosis of OSAHS. However, the test is costly and time taking, accessible in big cities only and is not always well accepted by patients. Therefore approximately $95 \%$ of the patients with sleep disorders are not diagnosed. Although subjective, the Epworth Sleepiness Scale may contribute to analyze signs and symptoms. It is easy to be applied, fast and free of charge. The purpose of our study was to evaluate Epworth Sleepiness Scale Scores and AHI in obese patients and to compare these parameters, Methods: The present study is an observational study which was conducted in the Department of Pulmonary Medicine, People's College of Medical Science \& Research Centre, Bhopal from Oct. 2013 to April 2015. Randomly selected 100 patients with BMI $\geq 23$ fulfilling inclusion and exclusion criteria were assessed in the study. Result: In our study we took 100 patients, 5 of them have ESS score $<9$ and 95 patients have ESS >9. In mild AHI group 2, patients were in ESS $<9$ group and 6 patients were in ESS $>9$ group. In moderate AHI group 2 patients were in ESS score $<9$ group and 25 patients were in ESS $>9$ group. In severe AHI group only 1 patient were in ESS $<9$ group and 64 patients were in ESS >9 group. Conclusion: We concluded that ESS shows correlation with AHI for severe and normal levels, but not for mild and moderate levels.
\end{abstract}

Key words: OSAHS, AHI, Epworth Sleepiness.

\section{Introduction}

Many patients search for medical care to treat snoring and excessive day time sleepiness. An appropriate workup can prove that snoring and excessive day time sleepiness may be a symptom of a sleep disorder, obstructive sleep apnea hypopnea syndrome (OSAHS), the prevalence of which is increasing in developed and developing countries [1]. Apnea Hypopnea Index is used to measure severity of OSAHS. It is classified as mild $(<5)$, moderate $(5-15)$, severe $(>15)$. Currently, overnight polysomnography is the gold standard for diagnosis of OSAHS. However, the test is expensive, time-consuming, of difficult access outside big cities and is not always well accepted by patients [2,3]. The aim of this study was to evaluate weather Excessive day time sleepiness measured by Epworth Sleepiness Scale

Manuscript received $11^{\text {th }}$ April 2016

Reviewed: $25^{\text {th }}$ April 2016

Author Corrected: $8^{\text {th }}$ May 2016

Accepted for Publication 24 $4^{\text {th }}$ May 2016
Scores can replace polysomnography for diagnosis of OSAHS.

\section{Methods}

The present study is an observational study conducted in the Department of Pulmonary Medicine People's College of medical Sciences \& Research Centre, Bhopal from Oct. 2013 to April 2015. 100 randomly selected patients with $\mathrm{BMI} \geq 23$ fulfilling inclusion and exclusion criteria were assessed in the study. All patients were registered after taking written informed consent.

They were explained about polysomnography and other investigations. Patients had full liberty to withdraw from this study without compromising his/her medical care as no intervention or treatment was involved. 
Every patient was given Epworth Sleepiness questionnaire [4], which consisted of eight questions. Each patient was asked to give points from 0 - 3 to each question on the basis of severity of their symptoms listed in question. Full confidentiality of data was maintained.

All religions and customs were respected. All these patients underwent overnight polysomnography using ALICE PDX polysomnography machine.

\section{Inclusion criteria}

1. Age 18-80 years of either sex.

2. BMI of more then or equal to 23

3. Informed and willing persons who had given consent for the study.

\section{Exclusion criteria}

1. Non consenting patients

2. Patients not tolerating sleep study.

3. Severe hypoxic patients

4. Patients with upper airway abnormality.

5. Patients with other comorbid conditions like coronary artery disease, bronchial asthma, chronic obstructive airway disease and malignancy.

6. Known hypothyroid patients.

Statistical analysis was done using Statistical Package of Social Science (SPSS Version 19; Chicago Inc., USA). Data comparison was done by applying specific statistical tests to find out the statistical significance of the comparisons.

\section{Results}

In this study 100 patients were taken, 5 of them had ESS score $<9$ and 95 patients had ESS $>9$. In ESS $<9$ group 2 patients and 6 patients were in ESS $>9$ group.

Table-1: Association of AHI with ESS.

\begin{tabular}{|c|c|c|c|c|}
\hline & \multicolumn{2}{|c|}{ ESS } & Total & Mean ESS \\
\hline AHI & ESS $<9$ & ESS $>\mathbf{9}$ & & 8 \\
\hline Mild $(<5)$ & 2 & 6 & 8 & 14 \\
\hline Moderate $(5-15)$ & 2 & 25 & 27 & 17 \\
\hline Severe $(>15)$ & 1 & 64 & 65 & \\
\hline Total & $\mathbf{5}$ & $\mathbf{9 5}$ & $\mathbf{1 0 0}$ & \\
\hline
\end{tabular}

In moderate AHI group 2 patients were in ESS score <9 group and 25 patients were in ESS >9 group. In severe AHI group only 1 patient was in ESS $<9$ group and 64 patients were in ESS $>9$ group. Mean ESS in mild AHI group was 8 which increases to moderate AHI group to 14 and in severe AHI group mean ESS was 17.

Above table shows sensitivity of ESS score is $75 \%$ in mild OSA which increases to $92.6 \%$ in moderate OSA which further increases to $98.5 \%$ in sever OSA.

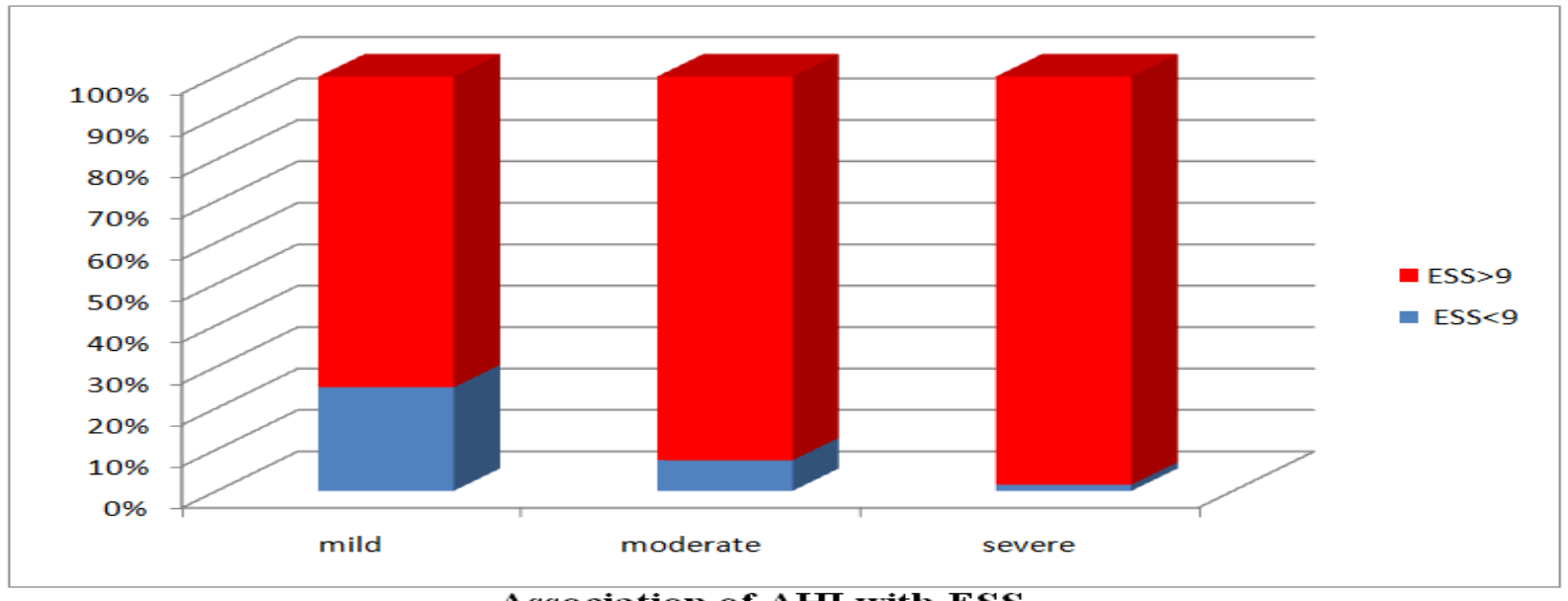

Association of AHI with ESS 


\section{Discussion}

According to American Academy of Sleep Medicine Obstructive Sleep Apnea and Hypopnea Syndrome is recurrent episodes of total or partial upper airways obstruction, causing desaturation and sleep fragmentation which is associated with the frequent symptom of excessive daytime sleepiness [5]. About $90 \%$ of patients affected with OSAHS show excessive daytime sleepiness, whereas the remaining patients show changes only during overnight monitoring [6].

Overnight polysomnography is accepted as the gold standard for OSAHS diagnosis. However, the test is expensive and of difficult access.

Epworth Sleepiness Scale was developed in 1991 by Dr. John W. Murray, since then, it has been used globally, and it was translated into many languages, such as German, Spanish and Japanese. but it is influenced by patient's reading and comprehension skills and honest answers. The Scale was able to separate primary snoring subjects from patients with OSAHS; the higher the ESS score, the more severe the condition. All patients with severe conditions scored over 10 [4]. However, it is worth noting that the severity level used by Dr. Murray was based on his judgment. In his study AHI $<5$ was normal; 5 to 25 was mild; 25 to 50 was moderate, and $>50$ was severe.

In our study symptom of excessive day time sleepiness was measured by Epworth sleepiness scale which has sensitivity $75 \%$ in mild OSA which increases to $92.6 \%$ in moderate OSA which further increases to $98.5 \%$ in severe OSA.

Similar results were found in the study done by Leticia Boari et al [7] who evaluated ESS score in 66 patients with OSAHS syndrome and found that $78 \%$ of patients with normal AHI scored less than 10 in the ESS and $65 \%$ of patients with severe AHI scored more than 10 . In groups presenting mild and moderate apnea no statistically significant result was found. Therefore they concluded that ESS can detect normal and severe levels of apnea, but is not able to detect mild and moderate levels and that ESS can be used in the follow-up of patients with OSAHS. Similarly, Bausmer U et al [8] tested for a possible correlation between ESS score and arithmetic values of AHI. No correlation between ESS and $\mathrm{AHI}$ was proven and therefore they concluded that it is not possible to predict solely on the basis of the ESS score the existence of OSA.
Rakan M. Haddad [9] et al also conduct a retrospective study of patients diagnosed to have obstructive sleep apnea syndrome. Epworth Sleepiness Scale score and Apnea-Hypopnea Index of 118 patients were compared and concluded that Epworth Sleepiness Scale was sensitive in patients with severe OSAS.

However, the accuracy of the ESS becomes less in mild and moderate OSAS.

Jianyin Zou et al [10] also conduct a study to develop a simple and efficient model for identifying OSA in Chinese adult population. They found that the ESS was moderately useful for screening undiagnosed OSA in adult population, and there may exist sex differences in ESS score cut-offs.

\section{Conclusion}

We concluded that symptom of excessive day time sleepiness as measured by ESS can help to predict severe OSA but not mild and moderate OSA. Therefore, it can be used as a diagnostic tool but should not replace polysomnography.

Funding: Nil, Conflict of interest: None initiated. Permission from IRB: Yes

\section{References}

1. Bittencourt LR, Togeiro SMGP, Bagnato MC. Diagnóstico da Síndrome da apnéia e hipopnéia obstrutiva do sono. In: Stamm A. ed. Rinologia; 2002; São Paulo: Komedi; 2002: 103-11.

2. Otorrinolaringológica em Cirurgia de Ronco e Apnéia. Revista Brasileira de Otorrinolaringologia set / out 2002; 68(5) supl.3.

3. Zonato AI, Bittencourt LR, Martinho FL, Júnior JFS, Gregório LC, Tufik S. Association of Systematic Head and Neck Physical Examination With Severity of Obstructive Sleep Apnea-Hypopnea Syndrome. Laryngoscope. 2003; 113(6):973-80.

4.Johns MW. A new method for measuring daytime sleepiness : the Epworth sleepiness scale. Sleep. 1991 Dec; 14 (6) : 540-5.

5.Sleep-Related Breathing Disorders in Adults: Recommendations for Syndrome Definition and 
Measurement techniques in Clinical Research- AASM Task Force. Sleep 1999; 22(5): 667-89.

6. Douglas NJ. The Obstructive Sleep Apnea/Hypopnea syndrome. Practical Neurology 2003; 3(1): 22-9.

7. Boari L, Cavalcanti C, Bannwart S, Sofia O, Dolci J. Avaliação da escala de Epworth em pacientes com a Síndrome da apnéia e hipopnéia obstrutiva do sono. Revista Brasileira de Otorrinolaringologia. 2004; 70 (6) : 752-756.

8. Bausmer U, Gouveris H, Selivanova O, Goepel B, Mann W (2010) Correlation of the Epworth Sleepiness
Scale with respiratory sleep parameters in patients with sleep-related breathing disorders and upper airway pathology. Eur Arch Otorhinolaryngol 267: 1645-164.

9. Haddad R, Al Sureehein S, Abu Alsamen G. Evaluation of Epworth Sleepiness Scale as a Screening Method for Obstructive Sleep Apnea Syndrome (OSAS). Middle East Journal of Internal Medicine. 2015; 8(2):3-6.

10. Zou J, Guan J, Yi H, Meng L, Xiong Y, Tang X et al. An Effective Model for Screening Obstructive Sleep Apnea: A Large-Scale Diagnostic Study. PLoS ONE. 2013; 8(12):e80704.

\section{How to cite this article?}

Tandon S, Joshi P, Nagdeote S. T. Obstructive sleep apnea and excessive day time sleepiness as measured by epworth sleepiness scale. Int J Med Res Rev 2016;4 (6):935-938doi: 10.17511/ijmrr.2016.i06.12. 